\title{
Supraglottic jet oxygenation and ventilation assisted fiberoptic intubation in a paralyzed patient with morbid obesity and obstructive sleep apnea: a case report
}

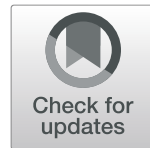

Hansheng Liang ${ }^{1}$, Yuantao Hou' ${ }^{1}$ Huafeng Wei ${ }^{2}$ and Yi Feng ${ }^{1 *}$ (D)

\begin{abstract}
Background: Hypoxia is a major concern and cause of morbidity or mortality during tracheal intubation after anesthesia induction in a pathological obese patient with obstructive sleep apnea (OSA). We introduce a case using Supraglottic jet oxygenation and ventilation (SJOV) to promote oxygenation/ventilation during fiberoptic intubation in a paralyzed patient with morbid obesity and OSA.

Case presentation: A 46-year-old man weighting $176 \mathrm{~kg}$ with BMI $53.7 \mathrm{~kg} / \mathrm{m} 2$ was scheduled for gastric volume reduction surgery to reduce body weight under general anesthesia. SpO2 decreased during induction, and two hand pressured mask ventilation partial failed. We then placed WEI Nasal Jet Tube (WNJ) in the patient's right nostril to provide SJOV. Then fiberoptic bronchoscopy guided endotracheal intubation was performed via mouth approach, and vital signs were stable. The operation was successfully completed after $3 \mathrm{~h}$. Patient recovered smoothly in hospital for 8 days and did not have any recall inside the operating room.

Conclusion: SJOV via WNJ could effectively maintain adequate oxygenation/ventilation during long time fiberoptic intubation in an apnea patient with morbid obesity and OSA after partial failure of two hand pressured mask ventilation, without obvious complications. This may provide a new effective approach for difficult airway management in these patients.
\end{abstract}

Keywords: Supraglottic, Jet ventilation, Oxygenation, Obesity, OSA, Fiberoptic bronchoscope, Intubation, Difficult airway

\section{Background}

The pathological difficult airway usually place patients in danger during general anesthesia induction [1], which may result in a high percentage of airway-related morbidity and mortality $[2,3]$. Hypoxia is a major concern and cause of morbidity or mortality during tracheal intubation after anesthesia induction in a pathological obese patient with obstructive sleep apnea (OSA) [4-6]. A new approach to promote oxygenation/ventilation has been described during various difficult airway managements (Peng J,Xie P,Wu CN,Wei HF,et al.) [1, 7-11] and under propofol anesthesia ( $\mathrm{Su} D S, B J A$ 2017), especially

\footnotetext{
* Correspondence: fengyimzk@163.com

'Department of Anesthesiology, Peking University People' s Hospital, Beijing 100044, China

Full list of author information is available at the end of the article
}

in obese patient. We introduce a case using Supraglottic jet oxygenation and ventilation (SJOV) to promote oxygenation/ventilation during fiberoptic intubation in a paralyzed patient with morbid obesity and OSA.

\section{Case presentation}

A 46-year-old man weighting $176 \mathrm{~kg}$ with BMI $53.7 \mathrm{~kg} /$ $\mathrm{m} 2$ was scheduled for gastric volume reduction surgery to reduce body weight under general anesthesia. The patient was diagnosed of obstructive sleep apnea (OSA) 3 years ago, without treatment. Airway inspection showed short neck with circumference of $51 \mathrm{~cm}$, limited neck extension due to its thick fat and the Mallampatti score-Ш. The patient felt tired preoperatively because of his sleep deprivation secondary to OSA. He was very 
nervous and refused to consent for awake fiberoptic intubation under sedation.

We elected to perform tracheal intubation after anesthesia induction but keeping patient's spontaneous breathing to avoid hypoxia, with initial direct laryngoscopy using video laryngoscope, and back up with fiberoptic intubation and then laryngeal mask airway (LMA). Bispectral index (BIS) was used to monitor anesthesia depth.

Vital signs showed Bp 142/79 mmHg, HR $88 \mathrm{bpm}$, SpO2 96\%, RR 22 bpm before anesthesia induction. Midazolam $3 \mathrm{mg}$ and sufentanil $10 \mu \mathrm{g}$ was given intravenously to reach BIS at 62 for sedation. Thereafter, intravenous $100 \mathrm{mg}$ propofol was given and BIS fell to 51. Mask pressurized ventilation could be performed to maintain $\mathrm{SpO} 2$ 100\% with patient under continuous target controlled infusion (TCI) at propofol $3 \mu \mathrm{g} / \mathrm{mL}$.Direct laryngoscope with video laryngoscope was tried twice but failed because of the invisible glottis obstructed by Huge epiglottis (Grace IIb view). SpO2 fell to $75 \%$ at the end of second laryngoscopy. Two hand pressurized mask ventilation was initiated and became difficult, although $\mathrm{SpO} 2$ could be maintained around $88 \%$ with following vital signs: BP 133/73 mmHg, HR $86 \mathrm{bpm}$, normal sinus rhythm, BIS 57.We then placed WEI Nasal Jet Tube (WNJ), (Well Lead Medical Equipment Ltd., Guangzhou, China. Production batch number: 20140901) (Fig. 1) in the patient's right nostril to provide SJOV. The jet catheter of the WNJ was connected to an automatical jet ventilator-TKR-400 (Well Lead Medical Equipment Ltd.,Guangzhou, China.) with following working parameters: driving pressure (DP) $35 \mathrm{psi}$, respiratory rate (RR): $55 \mathrm{bpm}, \mathrm{I} / \mathrm{E}$ ratio 1:3.SpO2 began to rise again and reached $100 \%$ at $1 \mathrm{~min}$ after initiation of SJOV. Thoracic cage moved ups and downs during SJOV indication of both oxygenation and ventilation. We then administered intravenous rocuranium $60 \mathrm{mg}$, TCI propofol $4 \mu \mathrm{g} / \mathrm{mL}$ and controlled ventilation was achieved using SJOV. Fiberoptic bronchoscopy guided endotracheal intubation was performed via mouth approach. Fiberoptic intubation was difficult due to hypertrophy of the patient's tongue and epiglottis but eventually succeded $5 \mathrm{~min}$ later. The vital signs at the end of successful intubation were as followings: SpO2 100\%,BP 125/64 mmHg, HR $71 \mathrm{bpm}$, sinus rhythm, BIS 45.PetCO2 was not monitored during fiberoptic intubation due to the both Ports being not consistent and hurry to raise oxygenation. However,instant blood gas analysis showed $\mathrm{pH} 7.36$, $\mathrm{PaO} 2124 \mathrm{mmHg}$ and $\mathrm{PaCO} 249 \mathrm{mmHg}$. Total time of SJOV via WNJ was about $7 \mathrm{~min}$. No obvious barotrauma, nose bleeding etc.,was seen at the end of intubation.

The operation was successfully completed after $3 \mathrm{~h}$. The patient was transferred to the ICU and was extubated in ICU without event. Patient recovered smoothly in hospital for 8 days and did not have any recall inside the operating room.

\section{Discussion and conclusion}

This case clearly demonstrated the SJOV via WNJ could effectively maintain adequate oxygenation/ventilation during long time fiberoptic intubation in an apnea patient with morbid obesity and OSA after partial failure of two hand pressured mask ventilation, without obvious complications. This may provide a new effective approach for difficult airway management in these patients.

According to the world health organization (WHO) standards established 1989 , body mass index (BMI) $\geqq 28$

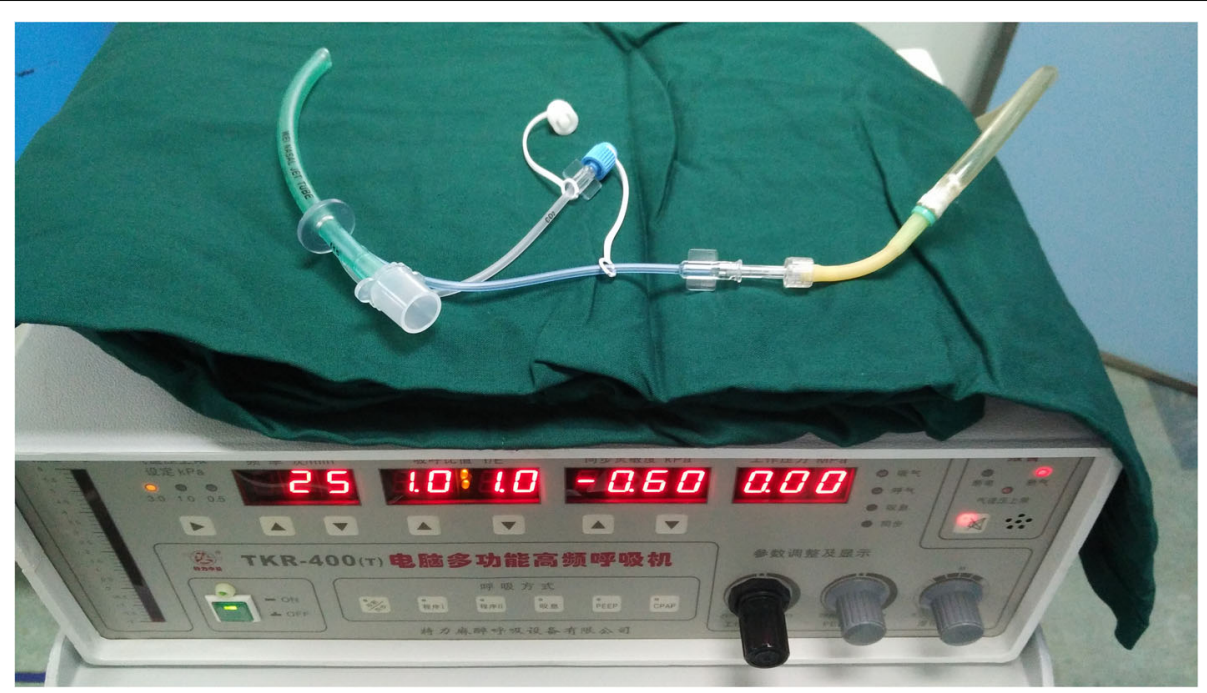

Fig. 1 Components and assembly of the WEI Nasal Jet Tube (WNJ) and automatical jet ventilator: $\mathrm{P}_{\mathrm{et}} \mathrm{CO}_{2}=$ end tidal $\mathrm{CO}_{2}$ pressure $(\mathrm{Compliance}$ with ethical standards: The automatical jet ventilator-TKR-400 and WNJT upon examination and approval by the company, pictures can be used) 
is defined as obesity, $\mathrm{BMI} \geqq 40$ is defined as morbid obesity [12]. Adipose tissue accumulated in pharyngeal can make patient's pharyngeal cavity narrow and make the oropharyngeal muscles and soft tissue collapse, obstructing the airway $[12,13]$. Obviously, this increases the patient's workload of breathing and reducing functional residual capacity, causing ventilation/perfusion mismatch,eventually hypoxia and intolerance of hypoxia [14]. The incidence of difficult intubation in morbid obesity patients during general anesthesia induction undergoing abdominal surgery can be as high as $24 \%$ [15], and the awake intubation may be required in about $8 \%$ of patients [15]. The potential upper respiratory tract obstruction in OSA patient contribute to its high perioperative complication rate at about $19.6 \%$ [16]. The incidence of failed intubation in OSA patients is 10 times higher than normal [17], while the incidence of intubation failure about patients with morbid obesity and OSA increases to 100 times [17, 18]. We discussed awake intubation with this patient on pre-anesthesia visit, but the patient was nervous and could not be comforted and refused the plan. In addition, the patient's large neck circumference makes it difficult to identify the cricothyroid membrane. Since we underestimated the difficulty of this patient's airway, and this patient was very nervous and unwilling to be stimulated, we did not choose awake intubation or awake look. Even though this case was an unanticipated difficult airway, we were able to maintain and improve oxygenation using WEI nasal jet tube for supraglottic jet oxygenation and ventilation,because we have some experiences applying this device on obese patients. We have completed a randomized controlled study(Registration number, ChiCTR $1,800,017,028)$ of supraglottic jet ventilation to improve oxygenation in obesity patients, and the results revealed that Nasal Jet tube could prevent patients suffering from desaturation during propofol-remifentanil protocol undergoing hysteroscopy. We are in the process of writing a paper.

The most important features of supraglottic airway is most characteristic of the patient with pathological obesity and OSA is serious airway collapse after anesthesia due to hypertrophy of the patient's tongue,epiglottis and supraglottic muscles [19]. Airway collapse make supraglottic airway narrower, making the air flow of the mask pressurized ventilation difficult to pass the narrow area [20]. WNJ can pass the narrow airway and support the collapse and resolve the ventilation problem. WNJ is designed to be soft to void injury of nasal mucosa damage and bleeding. The evaluation of nasal cavity and the use of the lubricant also ease its insertion and minimizing nose bleeding. In our case, nasal jet tube maintained this patient's oxygenation. According to the "Difficult Airway Society 2015 guidelines for management of unanticipated difficult intubation in adults" [21], muscle relaxant can make tracheal intubation easier, and prevent laryngospasm, so we administered rocuronium. Succinylcholine should be a better choice in this case, but we don't have it in our department. With quick and short-acting effect, rocuronium could exert muscle relaxant effect $60 \mathrm{~s}$ after administration, which is equivalent with succinylcholine.

The capacity of tolerance to hypoxia in pathological obese patients with OSA is reduced significantly. It is critical in this case to have an effective method to maintain the patient's oxygenation during intubation because of the shorter Oxygen Saturation Falling Time (OSFT) [22]. The definition of OSFT is the time of the patient's $\mathrm{SpO} 2$ dropped to $90 \%$ after full oxygenation $100 \%$ oxygen inhaled and removed nitrogen $[22,23]$. The obese patient's OSFT is only $163 \mathrm{~s}$ due to the increased oxygen consumption and the changes in the airway described above,but the average of a normal adult is $526 \mathrm{~s}$ [22-24]. So it is almost impossible to complete this fiberoptic intubation without maintaining effective oxygenation using SJOV via WNJ for 7 min in this anesthetized and paralyzed morbid obese patient with OSA. This patient didn't reach "can't intubate and can't ventilate" (CICV) so that LMA placement was not tried, nor did the emergent tracheotomy was considered.

In combination with previous publications [1, 7-11], It seems that SJOV has following advantages compared to the transtracheal jet ventilation (TTJV) recommended by American Society of Anesthesiologists (ASA) guidelines for the management of difficult airway. First,SJOV via WNJ is less invasive than TTJV which may increase the incidence of barotrauma barotrauma [7, 19]. Secondly, it usually take longer time to perform effective TTJV than placement of WNJ in the nose and could be very difficult to achieve successful TTJV in obese patient with thick neck. Thirdly, it is convenient to monitor ventilation using the built-in port for PetCO2 monitoring in WNJ. Forth and most importantly, SJOV maintain the open feature of airway during jet ventilation, minimizing or preventing the severe barotrauma, often seen during the use of TTJV, especially in emergent difficult airway management [1].

In summary, SJOV via WNJ seems to be an effective new approach to maintain adequate oxygenation/ventilation during difficult airway management in an apnea patient with morbid obesity and OSA.

\footnotetext{
Abbreviations

ASA: American Society of Anesthesiologists; BIS: Bispectral index; BMI: Body mass index; CICV: Can't intubate and can't ventilate; DP: Driving pressure; LMA: Laryngeal mask airway; OSA: Obstructive sleep apnea; OSFT: Oxygen Saturation Falling Time; SJOV: Supraglottic jet oxygenation and ventilation; TCl: Target controlled infusion; TTJ: Transtracheal jet ventilation; WHO: World health organization; WNJ: WEI Nasal Jet Tube
} 


\section{Acknowledgements}

Not applicable.

\section{Funding}

The authors declare that they have no funding.

\section{Availability of data and materials}

All attainable data are within in the paper and Fig.1. All data are fully available without restriction.

\section{Authors' contributions}

HSL, YTH, HFW and YF participated in data collection and analysis. HSL drafted the manuscript, YF designed the study. All authors approved the final version of the manuscript.

\section{Ethics approval and consent to participate}

A local ethics committee ruled that no formal ethics approval was required in this particular case.

\section{Consent for publication}

Written informed consent was obtained from the patient for publication.

\section{Competing interests}

Huafeng Wei is the inventor of the WEI Nasal Jet Tube (WEI NASAL JET or WNJ), which was used to generate SJOV in this study. The University of Pennsylvania initiated and granted the patent application for the WNJ in several countries, including the United States, China, Europe, Japan, Canada, Australia, and Brazil. The other authors have no potential competing interests to declare.

\section{Publisher's Note}

Springer Nature remains neutral with regard to jurisdictional claims in published maps and institutional affiliations.

\section{Author details}

'Department of Anesthesiology, Peking University People's sospital, Beijing 100044, China. ${ }^{2}$ Department of Anesthesiology and Critical Care, Hospital of the University of Pennsylvania, Philadelphia, PA 19104, USA

Received: 3 January 2019 Accepted: 5 March 2019

Published online: 20 March 2019

\section{References}

1. Peng J, Ye J, Wei H, et al. Supraglottic jet ventilation in difficult airway management. J Emerg Med. 2012;43(2):382-90.

2. Fritzsche K, Osmers A. Anesthetic management in laryngotracheal surgery. High-frequency jet ventilation as strategy forventilation during genera anesthesia. HNO. 2011;59(9):931-41.

3. Leiter R, Aliverti A, Frykholm P, et al. Comparison of superimposed highfrequency jet ventilation with conventional jet ventilation for laryngea surgery. Br J Anaesth. 2012;108(4):690-7.

4. Ross-Anderson DJ, Ferguson C, Patel A. Transtracheal jet ventilation in 50 patients with severe airway compromise and stridor. Br J Anaesth. 2011; 106(1):140-4.

5. Ihra GC, Tsai CJ, Kimberger O. Intrinsic positive end-expiratory pressure at various frequencies of supraglottic jet ventilation in a model of dynamic upper airway obstruction. Anesth Analg. 2010;111(3):703-6.

6. Cobas MA, Martin ND, Barkin HB. Two lost airways and one unexpected problem: undiagnosed tracheal stenosis in a morbidly obese patient. J Clin Anesth. 2016:35:225-7.

7. Li Q, Xie P, Wei H, et al. Supraglottic jet oxygenation and ventilation saved a patient with 'cannot intubate and cannot ventilate' emergency difficult airway. J Anesth. 2017;31(1):144-7.

8. Wu CN, Ma WH, Wei JQ, et al. Laryngoscope and a new tracheal tube assist Lightwand intubation in difficult airways due to unstable cervical spine. PLoSOne. 2015;10:202-31.

9. Wei HF. A new tracheal tube and methods to facilitate ventilation and placement in emergency airway management. Resuscitation. 2006;70:438-44.

10. Yang ZY, Meng Q, Xu YH, Wang JW, Yu DS, Wei HF. Supraglottic jet oxygenation and ventilation during colonoscopy under monitored anesthesia care: a controlled randomized clinical trial. Eur Rev Med Pharmacol Sci. 2016:20:1168-73.

11. Dziewit JA, Wei H. Supraglottic jet ventilation assists intubation in a Marfan's syndrome patient with a difficult airway. J Clin Anesth. 2011;23:407-9.

12. Mushambi MC, Kinsella SM, Popat M, et al. Obstetric Anaesthetists' Association and Difficult Airway Society guidelines for the management of difficult and failed tracheal intubation in obstetrics. Anaesthesia. 2015;70(11): 1286-306.

13. Troop C. The difficult airway and or obesity and the importance of positioning. Br J Anaesth. 2016;117(5):674-5

14. Dohrn N, Sommer T, Larsen JF, et al. Difficult Tracheal Intubation in Obese Gastric Bypass patients. Obes Surg. 2016;26(11):2640-7.

15. Whalen FX, Gajieo T, Hompson GB, et al. The effects ofthe alveolar recruitment maneuver and positive end-expiratory pressure on arterial oxygenation during laparoscopie bariatrie surgery. Anesth Analg. 2006; 102(1):298-305

16. Corso RM, Cattano D, Maitan S, et al. Post analysis simulated correlation of the El-Ganzouri airway difficulty score with difficult airway. Braz J Anesthesiol. 2016;66(3):298-303.

17. Stierer TL, Wfiight C, George A, et al. Risk assessment of obstructive sleep apnea in a population of patients undergoing ambulatory surgery. J Clin Sleep Med. 2010;6(5):467-72.

18. Gupata RM, Parvizl J. Postoperative complication in patients with obstructive sleep apnea syndrome undergoing hip or knee replacement:a case-control study. Mayo Clin Proc. 2001;76(9):897-904.

19. Sato S, Sato Y, Isono S, et al. Mask Ventilation during Induction of General Anesthesia: Influences of Obstructive Sleep Apnea. Anesthesiology. 2017; 126(1):28-38.

20. Domi R, Laho H. Anesthetic challenges in the obese patient. J Anesth. 2012; 26(5):758-65.

21. Frerk C, Mitchell VS, McNarry AF, et al. Difficult airway society 2015 guidelines for management of unanticipated difficult intubation in adults. $\mathrm{Br}$ Anaesth. 2015;115(6):827-48

22. Gray EL, McKenzie DK, Eckert DJ. Obstructive Sleep Apnea without Obesity Is Common and Difficult to Treat: Evidence for a Distinct Pathophysiological Phenotype. J Clin Sleep Med. 2017;13(1):81-8.

23. Sprung J, Whalley DG, Faleone T. The effects of tidal volume and respiratory rate on oxygenation and respiratory mechanics during laparoseopy in morbidly obese patient. Anesth Analg. 2003;97(1):268-74

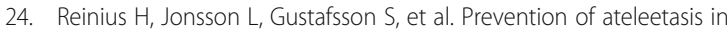
morbidly obese patients during general anesthesia and paralysis:a computerized tomography study. Anesthesiology. 2009;111(5):979-87.

\section{Ready to submit your research? Choose BMC and benefit from:}

- fast, convenient online submission

- thorough peer review by experienced researchers in your field

- rapid publication on acceptance

- support for research data, including large and complex data types

- gold Open Access which fosters wider collaboration and increased citations

- maximum visibility for your research: over $100 \mathrm{M}$ website views per year

At $\mathrm{BMC}$, research is always in progress.

Learn more biomedcentral.com/submissions 